\title{
Diferença entre intensidade do exercício prescrita por meio do teste TCAR no solo arenoso e na grama
}

\author{
Prescribed differences in exercise intensity based on the TCAR \\ test over sandy ground and grass
}

\author{
Tiago Cetolin \\ Valdeci Foza \\ Lorival José Carminatti 2 \\ Luiz Guilherme Antonacci Guglielmo ${ }^{3}$ \\ Juliano Fernandes da Silva
}

1. Faculdade da Serra Gaúcha, Escola Superior de Educação e Saúde, Caxias do Sul, RS. Brasil.

2. Universidade de Santa Catarina. Centro de

Ciências da Saúde e do Esporte. Laboratório de Pesquisa Morfo-funcional. Florianópolis, SC. Brasil.

3. Universidade Federal de Santa Catarina.

Centro de Desportos. Laboratório de Esforço Físico, Florianópolis, SC, Brasil.

Recebido em 14/11/08 Revisado em 05/12/08 Aprovado em 04/09/09
Resumo - A intensidade do treinamento pode ser influenciada pelo modo de exercício e tipo de terreno. Assim, os objetivos deste estudo foram: a) comparar os índices fisiológicos determinados no teste TCAR realizados no solo arenoso (SA) e no piso de grama natural (PGN) b) analisar as respostas de frequência cardíaca (FC) e lactato sanguíneo em exercício de carga constante em SA e PGN. Dez atletas de futebol $(15,11 \pm 1,1$ anos, $168 \pm 4,0 \mathrm{~cm}$, $60 \pm 4,0 \mathrm{~kg}$ ) foram submetidos ao teste TCAR para determinação do pico de velocidade (PV) e intensidade correspondente a 80,4\% do PV $($ V80,4) no PGN e no SA. A segunda avaliação consistiu de dois testes de carga constante (TCR) (80,4\%PV no PGN e no SA) com duração de 27 minutos. Foi utilizado o teste " $t$ " de Student pareado para comparar os testes no PGN e no SA. A análise de variância ANOVA (two-way), complementada pelo teste de Tukey foi utilizada para comparar os valores das concentrações de lactato [La], nos minutos 9, 18 e 27, nos dois tipos de solo. Foi adotado p<0,05. O PV e a V80,4 na grama $\left(15,3 \pm 1,0 \mathrm{~km} \cdot \mathrm{h}^{-1} \mathrm{e} 12,3 \pm 0,6 \mathrm{~km} \cdot \mathrm{h}^{-1}\right)$ foram significativamente superiores à areia $\left(14,3 \pm 1,0 \mathrm{~km} \cdot \mathrm{h}^{-1}\right.$ e $\left.11,5 \pm 0,4 \mathrm{~km} \cdot \mathrm{h}^{-1}\right)$. A concentração de lactato [laV80,4] no TCR da areia $\left(4,1 \pm 0,9 \mathrm{mmol} \cdot \mathrm{L}^{-1}\right)$ foi significativamente superior à grama $\left(2,8 \pm 0,7 \mathrm{mmol} . \mathrm{L}^{-1}\right)$. No TCR a FC média não apresentou diferença significativa, enquanto as [la] apresentaram, entre os dois terrenos. Pode-se concluir que o tipo de solo interfere nos indicadores associados à capacidade e à potência aeróbia, obtidos por meio do teste TCAR.

Palavras-chave: Treinamento; Capacidade aeróbia; Ciclo de velocidade.

Abstract - The intensity of training might be influenced by exercise mode and type of terrain. Thus, the objective of this study was a) to compare the physiological indices determined in the TCAR test carried out on natural grass (NG) and sandy ground (SG), and b) to analyze heart rate (HR) and blood lactate responses during constant exercise on SG and NG. Ten soccer players $(15.11 \pm 1.1$ years, $168 \pm 4.0 \mathrm{~cm}, 60 \pm 4.0 \mathrm{~kg}$ ) were submitted to the TCAR test to determine peak velocity (PV) and the intensity corresponding to 80.4\% PV (V80.4) on NG and SG. The second evaluation consisted of two constant load tests (CLT) (80.4\% PV on NG and SG) with a duration of $27 \mathrm{~min}$. The paired Student t-test was used to compare the tests carried out on NG and SG. ANOVA (two-way), complemented by the Tukey test, was used to compare lactate concentrations [La] at 9, 18 and 27 min between the two types of terrain. A p value $<0.05$ was adopted. PV and V80.4 $(15.3 \pm 1.0$ and $12.3 \pm 0.6 \mathrm{~km} / \mathrm{h})$ were significantly higher on grass than on sand $(14.3 \pm 1.0$ and $11.5 \pm 0.4 \mathrm{~km} / \mathrm{h})$. Lactate concentration during the CLT [LaV80.4] was significantly higher on sand $(4.1 \pm 0.9 \mathrm{mmol} / \mathrm{L})$ than on grass $(2.8 \pm 0.7 \mathrm{mmol} / \mathrm{L})$. In the CLT, no significant difference in mean $H R$ was observed between the two terrains, whereas there was a difference in [La]. In conclusion, the type of terrain interferes with indicators associated with aerobic power and capacity obtained by the TCAR test.

Key words: Training; Aerobic capacity; Peak velocity. 


\section{INTRODUÇÃO}

A intensidade do exercício representa uma das variáveis de maior impacto na prescrição do treinamento físico, em diversas modalidades esportivas, que apresentam características contínuas e intervaladas. Assim, para o controle da intensidade do treinamento, alguns índices fisiológicos têm sido utilizados, dentre os quais: o consumo máximo de oxigênio $\left(\mathrm{VO}_{2} \max \right)$, a intensidade mínima associada ao $\mathrm{VO}_{2} \max \left(\mathrm{IVO}_{2} \max \right)$, a máxima fase estável de lactato (MFEL) $)^{1}$ o pico de velocidade $(\mathrm{PV})^{2}$, o ponto de deflexão da frequência cardíaca (PDFC) $)^{3,4}$, o limiar ventilatório ${ }^{5}$, o percentual do $\mathrm{PV}^{6}$, o limiar de lactato $^{7}$ e a percepção subjetiva do esforço ${ }^{8}$.

Tradicionalmente, as avaliações dos índices fisiológicos citados são realizadas de forma contínua ${ }^{2,5,9}$. Porém, o perfil de atividades em modalidades esportivas como o futebol, envolve características intermitentes. Neste sentido, é crescente o número de estudos que procuram determinar indicadores fisiológicos de forma intermitente ${ }^{6,10,11}$. Um destes testes é o TCAR, proposto por Carminatti et al. ${ }^{11}$, para a avaliação da potência aeróbia a partir do PV e, posteriormente, validado para avaliação da capacidade aeróbia ${ }^{6}$, utilizando o PDFC e um percentual fixo $(80,4 \%)$ do PV. O TCAR contempla, na sua execução, mudança de sentido, acelerações e desacelerações, além de pausas entre os estímulos. O TCAR, além de ser utilizado para a obtenção de uma avaliação inicial da aptidão aeróbia dos atletas, permite que sejam transferidos os indicadores determinados para a prescrição do treinamento de forma específica ${ }^{6}$.

Um dos maiores problemas para a prescrição da intensidade de treinamento é a transferência dos indicadores obtidos em avaliação para a sessão de treino. Ahmaidi et al..$^{12}$ observaram que o teste de Léger ${ }^{9}$ apresentava validade para estimativa do $\mathrm{VO}_{2}$ max, porém, não era um bom indicador para determinação do PV, pois as suas constantes mudanças de sentido em espaço curto $(20 \mathrm{~m})$ impediam que os indivíduos alcançassem elevados valores de PV. Isso dificultava a utilização deste índice para a prescrição de treinamento da potência aeróbia.

O TCAR não apresenta esta limitação, pois o incremento na velocidade acontece em função do acréscimo na distância e não a partir da diminuição do tempo entre os sinais sonoros, apresentando valores mais elevados de PV quando comparados ao teste de Léger ${ }^{11}$. A adoção de pausas durante o protocolo é outro fator que deve ser considerado para uma posterior prescrição de treino, pois sucessivas pausas durante o protocolo podem contribuir para valores mais elevados nos indicadores fisiológicos ${ }^{13}$.

Outro ponto que deve ser considerado para a prescrição do treinamento é o tipo de terreno que o exercício é realizado. Estudos têm demonstrado que o terreno influencia diretamente na intensidade do trabalho ${ }^{14,15}$. Em atletas de futebol de campo observou-se uma diferença na velocidade de corrida em gramado seco e molhado ${ }^{16}$. A literatura tem sugerido que esse fenômeno também ocorre quando analisada a intensidade entre solos duros e arenosos ${ }^{15,17-19}$. Observando os resultados apontados pela literatura e o conhecimento da prática tradicional de treinamento físico em solo arenoso (SA), é fundamental que sejam realizados estudos investigando as possíveis diferenças na solicitação fisiológica em diferentes terrenos.

Sannicandro ${ }^{19}$, estudando atletas beach soccer, observou que o SA apresenta uma exigência 15\% maior ao terreno com uma maior compactação. Isto indica que parece haver uma exigência mais elevada no SA em comparação ao piso de grama, mais compacto. No entanto, ainda não foram investigadas as diferentes solicitações de acordo com o terreno utilizado, em testes intermitentes, específicos para modalidades coletivas.

Considerando os resultados apresentados na literatura, indicando que o tipo de terreno parece influenciar na demanda metabólica durante a realização do teste, pode-se propor que os índices fisiológicos determinados no TCAR (PV e 80\%PV) apresentariam menores valores no SA do que em piso de grama natural (PGN).

Desta forma, o presente estudo teve por objetivos: a) determinar e comparar os índices fisiológicos de potência e capacidade aeróbia determinados no teste intermitente TCAR, realizados em SA e PGN b) analisar as respostas de FC e lactato sanguíneo, durante o exercício retangular (80,4\% PV) , com duração de 27 minutos, em SA e PGN.

\section{PROCEDIMENTOS METODOLÓGICOS}

Participaram deste estudo dez jovens atletas de futebol do sexo masculino, pertencentes às categorias juvenil e infantil, de um clube profissional do Rio Grande do Sul (15,1 $\pm 1,1$ anos, $168 \pm 0,4 \mathrm{~cm}$, $60 \pm 4,0 \mathrm{~kg}$ ), com atividades sistemáticas de três a cinco sessões semanais, com três horas de duração. O termo de consentimento livre e esclarecido foi assinado pelos representantes legais dos atletas. Os procedimentos foram aprovados pelo Comitê de 
Ética da Universidade Federal de Santa Catarina (Protocolo 384/07).

Durante duas semanas, os participantes foram submetidos a testes de campo incremental e de carga constante. Inicialmente, distribuíram-se os atletas em dois grupos (G1 e G2), com cinco integrantes cada. O G1 realizou, primeiramente, os testes em solo de grama e depois em SA e o G2 realizou os testes em ordem inversa ao G1. Na segunda semana, realizaram-se os testes de carga constante, organizando os grupos conforme o teste incremental. Desta forma, buscou-se eliminar o fator de interferência de ordem dos testes. É importante ressaltar que os atletas utilizaram chuteiras para realização do teste na grama, enquanto que na areia realizaram as avaliações com os pés descalços. No total, cada indivíduo foi submetido a quatro avaliações, com intervalo mínimo de 48 horas.

\section{Teste Incremental TCAR}

Inicialmente, foi realizado o teste $\operatorname{TCAR}^{11,20}$ para determinar: frequência cardíaca máxima (FCmáx), concentração de lactato sanguíneo máxima ([Lamáx]) e PV. O TCAR é caracterizado por ser do tipo intermitente escalonado, com multiestágios de 90 segundos de duração, em sistema "ida-e-volta", constituído de 5 repetições de 12 segundos de corrida (distância variável), intercaladas por 6 segundos de caminhada. O ritmo é ditado por sinais sonoros (bip), em intervalos regulares de 6 segundos. $\mathrm{O}$ teste inicia com velocidade de $9,0 \mathrm{kmh}^{-1}$ (distância inicial de $15 \mathrm{~m}$ ), com incrementos de $0,6 \mathrm{~km} \cdot \mathrm{h}^{-1}$ a cada estágio, até a exaustão voluntária, mediante aumentos sucessivos de $1 \mathrm{~m}$ a partir da distância inicial $^{11}$ (figura 1). O PV determinado no TCAR apresentou elevada reprodutibilidade em um estudo com 24 atletas de futebol (coeficiente de correlação intraclasse $=0,93$ ) (dados não publicados); além disso, está associado $(r=0,74, p<0,01)$ com a velocidade referente ao consumo máximo de oxigênio (vVO$\left.{ }_{2} \max \right)$, mensurada em esteira rolante, em jogadores de futebol $(n=28)$ (dados não publicados).

O teste foi realizado em dois tipos de solo:

1) Arenoso, caracterizado como areia fina de água doce com aderência e sustentação inferior ao solo de grama, porém, superior ao tipo médio de SA de água doce.

2) Grama natural.

A FC foi monitorada durante todo teste, sendo que no final de dois estágios o participante realizava uma pausa de 1 minuto para as coletas de sangue.

Teste de carga retangular (TCR)

Após o TCAR, calculou-se a velocidade e a [la] correspondente a $80,4 \%$ do PV $^{6,21}$ através de interpolação linear, utilizando uma concentração acima e outra abaixo de $80,4 \%$. Foi adotado este valor, pois esta intensidade geralmente corresponde a MFEL no modelo intermitente do $\mathrm{TCAR}^{6}$. Por meio das velocidades obtidas, realizou-se um teste retangular (TCR) com o propósito de verificar a estabilidade do lactato sanguíneo nessas intensidades. O TCR foi composto de três séries de corrida de nove minutos, com um intervalo de 1 minuto para coleta da FC e do sangue. O TCR teve a mesma dinâmica (duas corridas, uma pausa) do teste incremental, porém, em uma intensidade constante. $\mathrm{O}$ ritmo foi ditado por um sinal sonoro (bip), em intervalos regulares de 6 segundos.

\section{Freqüência cardíaca e lactato sanguíneo}

A FC foi registrada com cardiofrequencímetros da marca Polar Electro ${ }^{\circledR}$, modelo FS1. As amostras de sangue foram coletadas do lóbulo da orelha $(25 \mu \mathrm{L})$ e em seguida, armazenadas em tubos eppendorfs, contendo $50 \mu \mathrm{L}$ de fluoreto de sódio $1 \%$, sendo, posteriormente, analisadas por meio do analisador eletroquímico modelo YSI 2700 STAT.

\section{Análise Estatística}

Foi utilizada a estatística descritiva (média e desviopadrão). Verificou-se normalidade na distribuição dos dados por meio do teste de Shapiro Wilk. O teste " $\mathrm{t}$ " de Student para amostras dependentes foi utilizado para comparar os valores de PV, V80,4, FCmáx, [laV80,4] e [lamáx] determinados na grama com aqueles obtidos na areia. Para comparação dos valores das [La], nos minutos 9, 18 e 27, nos dois tipos de solo (grama e areia), foi utilizada a análise de variância ANOVA (two-way),

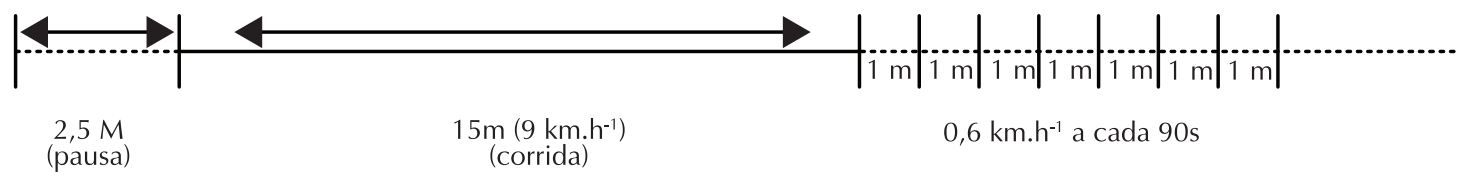

Figura 1. Visualização do esquema do teste intermitente TCAR. 
complementada pelo teste de Tukey. Adotou-se o nível de significância de 5\% ( $<<0,05)$.

\section{RESULTADOS}

Na tabela 1, são apresentados os valores das variáveis investigadas. Não foi encontrada diferença significativa $(p>0,05)$ na FCmáx do TCAR em ambos os solos. Este fato demonstra que os participantes atingiram elevados patamares de exigência cardíaca, em ambos os solos, que se aproximaram da FCmáx predita (220-idade).

Também não foram observadas diferenças ( $p>0,05)$ na [lamáx] entre os dois tipos de solo, porém, a média apresentou tendência a ser mais elevada no SA, o que sugere uma maior exigência metabólica para a realização do trabalho na areia. O percentual de diferença nos valores do PV, da V80,4 e da [laV80,4], entre os dois terrenos, foi 7\%, $7 \%$ e $46 \%$, respectivamente (tabela 1 ).

Tabela 1. Valores médios das respostas fisiológicas mensuradas no solo arenoso e de grama.

\begin{tabular}{|c|c|c|c|}
\hline Variável $(n=10)$ & Média & $\begin{array}{l}\text { Desvio } \\
\text { padrão }\end{array}$ & $\begin{array}{l}\text { \% dife- } \\
\text { rença }\end{array}$ \\
\hline FCmáx grama (bpm) & 198 & 10 & \multirow[b]{2}{*}{-} \\
\hline FCmáx areia (bpm) & 198 & 6 & \\
\hline [lamáx] grama (mmol.L-1) & 8,4 & 2 & \multirow[b]{2}{*}{20} \\
\hline [lamáx] areia $\left(\mathrm{mmol} . \mathrm{L}^{-1}\right)$ & 10,1 & 2 & \\
\hline PV grama $\left(K m \cdot h^{-1}\right)$ & $15,3^{\mathrm{a}}$ & 1 & \multirow{2}{*}{7} \\
\hline $\mathrm{PV}$ areia $\left(\mathrm{Km} \cdot \mathrm{h}^{-1}\right)$ & 14,3 & 1 & \\
\hline V80,4 grama $\left(\mathrm{Km} \cdot \mathrm{h}^{-1}\right)$ & $12,3^{\mathrm{a}}$ & 0,6 & \multirow[t]{2}{*}{7} \\
\hline V80,4 areia $\left(\mathrm{Km} \cdot \mathrm{h}^{-1}\right)$ & 11,5 & 0,4 & \\
\hline$\left[\right.$ laV80,4] grama $\left(\mathrm{mmol} . \mathrm{L}^{-1}\right)$ & $2,8^{\mathrm{a}}$ & 0,7 & \multirow{2}{*}{46} \\
\hline$\left[\right.$ laV80,4] areia $\left(\mathrm{mmol}^{\left.-\mathrm{L}^{-1}\right)}\right.$ & 4,1 & 0,9 & \\
\hline
\end{tabular}

${ }^{a} \mathrm{p}<0,05$ em relação a areia.

Nota: FCmáx = freqüência cardíaca máxima, [lamáx] = valor mais alto de lactato mensurado após o esforço, $\mathrm{PV}=$ pico de velocidade, $\mathrm{V} 80,4$ = velocidade correspondente a $80,4 \%$ do $\mathrm{PV},[\mathrm{laV} 80,4]=$ concentração de lactato correspondente a $80,4 \%$ do PV.

Na tabela 2, são apresentados os resultados das [la], obtidos no TCR para aproximação da MFEL, nas duas superfícies estudadas. Não foi encontrada diferença significativa $(\mathrm{p}>0,05)$ na FC entre as duas situações, demonstrando exigências similares do sistema cardiovascular nos dois tipos de solo. Porém, houve diferença $(\mathrm{p}<0,05)$ na [la], o que sugere uma maior exigência metabólica no SA.
Tabela 2. Valores médios e percentual de diferença para as variáveis FC e [la] na V80,4 nos dois tipos de solos.

\begin{tabular}{lcc}
\hline Variável $(\mathrm{n}=10)$ & Média \pm DP & \% diferença \\
\hline FC grama $(\mathrm{bpm})$ & $183 \pm 9$ & \\
FC areia $(\mathrm{bpm})$ & $185 \pm 10$ & - \\
[la] grama $\left(\mathrm{mmol} . \mathrm{L}^{-1}\right)$ & $3,3 \pm 0,9$ & 52 \\
[la] areia $\left(\mathrm{mmol} . \mathrm{L}^{-1}\right)$ & $5,1 \pm 1,0^{*}$ & \\
\hline
\end{tabular}

${ }^{*} \mathrm{p}<0,05$ em relação a [la] na grama.

Nota: FC grama = freqüência cardíaca mensurada no TCR na grama, $\mathrm{FC}$ areia $=$ freqüência cardíaca mensurada no TCR na areia, [la] grama = concentração de lactato mensurada no TCR na grama, [la] areia = concentração de lactato mensurada no TCR na areia.

Estão apresentados na figura 2 os valores médios referentes às [La] nos minutos 9, 18 e 27 no TCR nos dois terrenos, além da variação entre os minutos 9 e 27.

Foi possível observar que a variação nas [La] foi de 0,1 mmol. $\mathrm{L}^{-1}(3,4 \pm 0,5 ; 3,2 \pm 0,7$ e 3,5 $\pm 1,0$ mmol. $\left.\mathrm{L}^{-1}\right)$ na grama, enquanto que na areia foi de

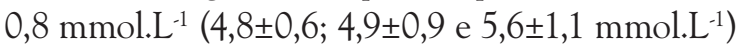
entre os minutos 9 e 27 .

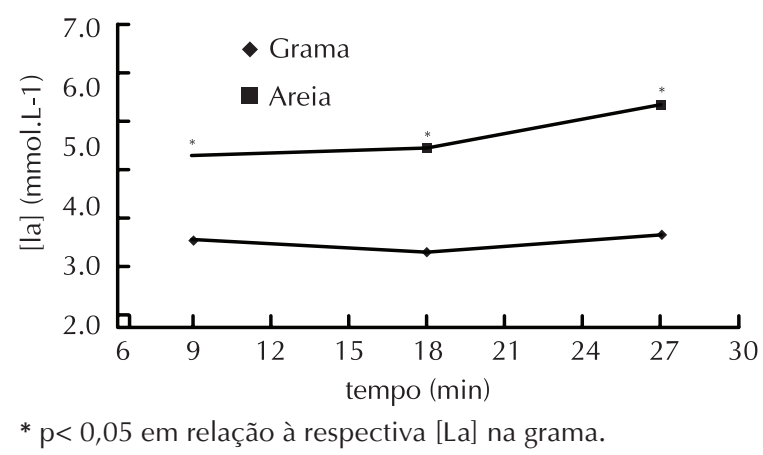

Figura 2. Valores médios de [La], nos minutos 9, 18 e 27, no teste de carga retangular.

\section{DISCUSSÃO}

principal achado deste estudo foi que os distintos tipos de solo acarretam diferentes solicitações metabólicas, que impossibilitam a transferência de indicadores obtidos em terreno compacto (grama) para o terreno arenoso, pois um mesmo valor absoluto de velocidade apresenta diferentes exigências fisiológicas de acordo com o terreno.

A intensidade de $80,4 \%$ do PV do TCAR foi escolhida para verificar as possíveis diferenças de solicitação metabólica entre grama e areia, em função desta ter sido validada como a velocidade correspondente a MFEL no TCAR 6 . Desta forma, trata-se da intensidade ótima para o aprimoramento da capacidade aeróbia ${ }^{13}$, podendo ser sustentada por 30 a 55 minutos em velocidade constante. 
No TCAR, os resultados demonstraram que o PV foi $7 \%$ menor no SA. Isto parece acontecer pela instabilidade e pela menor resistência oferecida pela areia, gerando uma sobrecarga na articulação do tornozelo, no momento de empurrar, ao longo do eixo vertical do movimento ${ }^{21}$. $\mathrm{O}$ indivíduo, com isso, tenta equilibrar e igualar o movimento, movendo o quadril a uma extensão maior ${ }^{22}$. Desta forma, este maior esforço contribui para menores valores de PV na areia.

Este valor de 7\%, ao comparar dois terrenos, é inferior ao encontrado por Sannicandro ${ }^{19}$. Porém, este estudo comparou a intensidade de exercício em um terreno de areia fina de água doce com PGN, enquanto Sannicandro ${ }^{19}$ utilizou SA de água salgada e, possivelmente, menos compacto que o SA deste estudo. Esse fato demonstra que diferentes tipos de intensidades são exigidos em cada classificação de solos arenosos (fino, médio e grosso; de água doce ou água salgada).

Nos dois testes incrementais, não foram encontradas diferenças significativas na lamáx. Isto demonstra que em situações de esforço máximo a [la] não parece ser diferenciada a partir do tipo de terreno utilizado.

No entanto, ao serem analisadas as intensidades submáximas, a resposta metabólica parece ser altamente dependente do padrão de movimento utilizado e, consequentemente, do tipo de terreno. Em atividades realizadas na areia há, entre outros fatores, um menor aproveitamento na energia elástica $^{17}$, que contribui para o aumento da intensidade relativa de esforço e um desgaste cumulativo em atividades de longa duração, apresentando uma maior solicitação metabólica quando comparada com a grama. Isto foi evidenciado no presente estudo, pois, mesmo a velocidade submáxima (V80,4\%) na areia $\left(11,5 \mathrm{~km} \cdot \mathrm{h}^{-1}\right)$ sendo menor que na grama (12,3 km.h-1 $\left.{ }^{1}\right)$, em termos absolutos, a corrida na areia ocasionou um estresse metabólico maior.

Estudos têm demonstrado a existência de um coeficiente de exigência que varia entre 1,2 a 1,6 a mais na solicitação energética, quando comparados solo de concreto e arenoso ${ }^{15,17,18}$. Os dados deste estudo apresentaram um valor $52 \%$ mais elevado de intensidade metabólica no teste retangular, realizado em SA, em comparação à grama. Esse valor sugere um coeficiente de 0,5 , porém, foi comparado um solo de grama com um arenoso, sendo que o PGN já tende a apresentar uma maior exigência metabólica do que o solo de concreto utilizado pelos autores ${ }^{15,17,18}$.

A explicação para a maior exigência do sistema metabólico no SA parece ser devido a um número maior de movimentos, como flexão de quadril e joelho, no início do contato do pé com areia ${ }^{18}$, que aumentam a solicitação metabólica em relação à grama. Desta forma, uma mesma intensidade absoluta não deve ser empregada com os mesmos objetivos, quando se utiliza os dois terrenos diferentes (grama e areia), pois ocasionarão respostas fisiológicas distintas.

Outro fator que pode estar envolvido na maior exigência metabólica no SA diz respeito a um aumento do tempo de contato do pé com o solo, uma degradação do potencial de energia elástica e da redução da eficiência completa do músculotendão ${ }^{15,17,23}$. Essa diminuição da eficiência conduz diretamente a uma redução da força ou energia do tornozelo para mover-se ao longo do eixo vertical do corpo, em comparação à corrida em superfície firme ${ }^{24}$. Na corrida em superfícies firmes, a energia elástica parece ser reutilizada nos músculos extensores da perna para aumentar a eficiência do movimento, tendo em contraponto que na corrida, em $\mathrm{SA}$, os movimentos sofrem perda de energia, principalmente, na extensão da articulação talocrural, encontrada na diferença de força aplicada ${ }^{24}$.

Assim, o trabalho mecânico exigido para correr na areia é mais elevado devido ao pé não deslizar e sim mover a areia, trazendo consigo um trabalho externo adicional, fato não observado na grama ${ }^{15}$. A penetração máxima do pé correndo foi calculada em $78 \%$ da profundidade da areia, acontecendo no término do passo, quando, com isso, as forças já estão reduzidas e são dirigidas, principalmente, horizontalmente ${ }^{15}$.

Portanto, a locomoção em SA demandaria uma maior resposta metabólica geral, já que em uma superfície dura, todo o trabalho positivo em uma fase de um passo é absorvido pelos músculos e tendões em uma fase subsequente. Parte dessa energia absorvida pode, durante o passo seguinte, aumentar a eficiência global ${ }^{15}$.

Em adição, a dificuldade de locomoção proporcionada pela superfície de areia tende a responder com uma eletromiografia aumentada nos músculos vasto medial e lateral, reto femoral, tensor da fáscia lata, semimembranoso e bíceps femoral, parecendo ser um dos fatores do maior gasto metabólico, quando correndo em areia em comparação à superfície de madeira ${ }^{21}$.

Outro fator diz respeito a um maior acionamento de unidades motoras glicolíticas anaeróbias para a realização do trabalho, o que poderia explicar, também, os valores mais elevados da [La], pois foi encontrado um percentual de diferença entre os solos de $52 \%$ no teste retangular. Isso permite 
inferir que a intensidade de $80,4 \%$ do $\mathrm{PV}$, na areia, apresenta uma solicitação metabólica diferenciada $(\mathrm{p}<0,05)$ em relação à grama. Desta forma, o limite superior de aprimoramento da capacidade aeróbia na areia parece ocorrer em percentuais diferentes do PV, nos dois terrenos. Os resultados indicam que existe a necessidade de um coeficiente de correção da intensidade, obtida por meio do teste incremental, para aplicação em treinos com intensidade constante e com longa duração em SA. Isto pode ser fundamentado em função da maior diferença percentual na [La], no teste retangular $(52 \%, p<0,05)$, em comparação ao incremental (20\%, p >0,05), ambos realizados nas duas condições (SA e PGN). Essa correção, talvez seja necessária devido à contínua exigência da manutenção de um padrão motor do exercício, à produção de potência por unidade de massa do músculo e ao tipo de fibra empenhada no exercício de longa duração ${ }^{24}$.

Desta forma, o trabalho realizado em SA, prescrito por meio de um teste incremental intermitente, tende a superestimar a intensidade em atividades de longa duração, devido a uma constante perda de eficiência e a um envolvimento progressivo de unidades motoras necessárias para o movimento.

\section{CONCLUSÃO}

Concluiu-se que o tipo de solo (arenoso e grama) interfere nos indicadores associados à capacidade e à potência aeróbia, obtidos por meio do teste incremental intermitente de campo, para modalidades coletivas (TCAR). Além disso, a transferência da V80,4 para prescrever o treinamento de média e longa duração, em solo arenoso, tende a superestimar a intensidade do exercício determinada na grama, sugerindo que seja necessária a elaboração de uma constante de correção.

\section{REFERÊNCIAS BIBLIOGRÁFICAS}

1. Beneke R. Maximal lactate steady state concentration (MLSS): experimental and modelling approaches. Eur J Applied Physiol 2003;88(4-5):361-369.

2. Noakes TD. Implications of exercise testing for prediction of athletic performance: a contemporary perspective. Med Sci Sport Exerc 1988;20(4):319-330.

3. Conconi F, Ferrari M, Ziglio PG. Determination of the anaerobic threshold by a noninvasive field test in runners. J Appl Physiol 1982;52(4):869-873.

4. Kara M, Gökbel H, Bediz C, Ergene N, Üçok K, Uysal H. Determination of the heart rate deflexion point by the Dmáx method. J Sport Med Phys Fitness 1996;36(1):31-34.
5. Loat CE, Rhodes EC. Relationship between the lactate and ventilatory threshold during prolonged exercise. Sport Med 1993;15(2):104-115.

6. Carminatti LJ. Validade de limiares anaeróbios derivados do teste incremental de corrida intermitente (TCAR) como preditores do máximo steady- state de lactato em jogadores de futsal. Dissertação de Mestrado - Programa de Pós-Graduação em Educação Física]. Florianópolis (SC): Universidade do Estado de Santa Catarina; 2006.

7. Farrel PA, Wilmore JH, Coyle EF, Billing JE, Costil DL. Plasma lactate accumulation and distance running performance. Med Sci Sport Exerc 1979;11(4):338-344.

8. Borg G, Noble B. Perceived exertion. Exer Sports Sci Rev 1974;2:131-153.

9. Léger LC, Lambert J. A maximal multistage 20-m shuttle run test to predict VO2max. Eur J Appl Physiol 1982;49(1):1-12.

10. Bangsbo J, Iaia FM, Krustrup P. The Yo-Yo intermittent recovery test a useful tool for evaluation of physical performance in intermittent Sports. Sport Med 2008;38(1):1-15.

11. Carminatti LJ, Lima-Silva AE, De-Oliveira FR. Aptidão Aeróbia em Esportes Intermitentes - Evidências de validade de construto e resultados em teste incremental com pausas (Resumo). Rev Bras Fisiol Exerc 2004; 3(1),p.120.

12. Ahmaidi S, Collomp K, Caillaud C, Préfaut, C. Maximal and functional aerobic capacity as assessed by two graduated field methods in comparison to laboratory exercise testing in moderately trained subjects. Int J Sport Med 1992;13(3):243-248.

13. Beneke R, Hütler M, Duvillard SPV, Sellens M, Leithäuser RM. Effect of test interruptions on blood lactate during constant workload testing. Med Sci Sports Exerc 2003; 35(9):1626-1630.

14. Lyons M, Al-Nakeeb Y, Nevill A. Performance of soccer passing skills under moderate and high-intensity localized muscle fatigue. J Strength Cond Res 2006;20(1):197-202.

15. Lejeune TM, Willems PA, Heglund NC. Mechanics and energetics of human locomotion on sand. J Exp Biol 1998; 201(13):2071-2080.

16. Moreira GMD. A influência de um gramado molhado sobre o desempenho em um teste progressivo para jogadores de futebol. [Dissertação de Mestrado - Programa de Pós Graduação em Educação Física]. Caxias do Sul (RS): Universidade de Caxias do Sul 2001.

17. Zamparo P, Perini R, Orizio C, Sacher M, Ferretti G. The energy cost of walking or running on sand. Eur J Applied Physiol 1992;65(2):183-187.

18. Pinnington H, Dawson B. Running economy of elite surf iron men and male runners, on soft dry beach sand and grass. Eur J Applied Physiol 2001;86(1):62-70.

19. Sannicandro I, Colella D, Anna Rosa R, Morano M. Motion skills on soft sand and conventional surface in beach soccer (Abstract). J Sport Sci Med 2007; 6(sup 10), p.169. 
20. Carminatti LJ, Lima-Silva AE, De-Oliveira FR. Dmáx em teste intermitente de campo: Estimativa por \% fixo da freqüência cardíaca máxima e pico de velocidade (Resumo). Anais do XXVIII Simpósio Internacional de Ciências do Esporte. São Paulo: 2005, p.44.

21. Pinnington HC, Lloyd DG, Besier TF, Dawson, B. Kinematic and electromyography analysis of submaximal differences running on a firm surface compared with soft, dry sand. Eur J Appl Physiol 2005;94(3):242-253.

22. Giatsis G, Kollias I, Panoutsakopoulos V, Papaiakovou G. Biomechanical differences in elite beach-volleyball players in vertical squat jump on rigid and sand surface. Sports Biomech 2004;3(1):145-158.

23. Morgan DL, Proske U. Factors contributing to energy storage during the stretch- shortening cycle. J Appl Biomech 1997;13(4):464-466.
24. Muramatsu S, Fukudome A, Miyama M, Arimoto M, Kijima A. Energy Expenditure in Maximal Jumps on Sand. J Physiol Anthropol 2006:25(1):59-61.

\section{Endereço para correspondência}

Juliano Fernandes da Silva

Universidade Federal de Santa Catarina

Centro de Desportos - Laboratório de Esforço Físico

Campus Universitário - Trindade

CEP: 88040-900 Florianópolis (SC) Brasil

E-mail: Jufesi23@yahoo.com.br 